\title{
Einleitung: Paronyme empirisch neu beleuchtet
}

\begin{abstract}
Der Beitrag führt in das Sonderheft „Paronymie im deutschen Sprachgebrauch“ ein und bündelt gewonnene Einblicke in die lexikologische, korpusanalytische sowie lexikografische Arbeit des Projektes „Paronymwörterbuch“. Er stellt wichtige Erkenntnisse zu Paronymen, ihrem Vorkommen, ihrer Ermittlung und Darstellung, aber auch zu den Methoden der Bedeutungsanalyse, ihren diskursiven Funktionen und dem Umgang mit Verwechslungspotenzial zusammenfassend dar. Vorgestellt werden Forschungsergebnisse, die sich vor allem auf die Verwendung von Paronymen in der öffentlichen Gebrauchssprache beziehen. Aber auch explorative Korpusverfahren werden erläutert sowie innovative, dynamische e-lexikografische Darstellungen präsentiert. Ausgewählte Probleme, die sowohl im Kontext der theoretischen Auseinandersetzung als auch mit der redaktionellen Erfassung von Paronymen auftreten, werden hier diskutiert. Das Sonderheft verbindet dabei theoretische und praktische lexikografische Herangehensweisen an ein bisher linguistisch wenig dokumentiertes Phänomen, das Muttersprachler/innen und Fremdsprachenlernende gleichermaßen verunsichert. Neben den Rückblicken werden ebenso die Ausblicke auf die kommenden Jahre und die damit verbundenen Fragestellungen des Projektes skizziert.
\end{abstract}

This paper is an introduction to the special issue "Paronymie im deutschen Sprachgebrauch" ("Paronymy in German usage') and brings together the results of the lexicological, corpus-analytical and lexicographic studies undertaken in the framework of the Paronym Dictionary project. It summarises important findings from its recent research on confusable terms, their occurrence, their corpus detection and their presentation, as well as methods of semantic analysis, information on their discursive textual functions and ways of dealing with the potential for lexical confusion. The paper presents research results, mainly on the use of paronyms in public language, explains explorative corpus methods and outlines innovative, dynamic e-lexicographic methods of documentation. It also discusses specific problems, particularly those which have occurred both in the context of theoretical discussion and of the editorial description of paronyms. This special issue sketches theoretical and practical lexicographic approaches to a phenomenon which has been largely neglected in linguistics, but one which causes uncertainty among native speakers and foreign language learners alike. Besides reviewing our research results, the paper also discusses future prospects and further open research questions for the project.

\section{Einführung}

Verwechselbare Ausdrücke gibt es zahlreiche. Bei näherer Betrachtung handelt es sich bei diesen Ausdrücken aber um sehr unterschiedliche lexikalische Einheiten bzw. um unterschiedliche linguistische Phänomene des sprachlichen Zweifels. Zu dieser heterogenen Gruppe werden beispielsweise Quasi-Synonyme gezählt (z. B. Grippe/Erkältung, kalt) kühl), Dubletten (webte/wob, gesendet/gesandt), Homofone (Föhn/Fön), falsche Freunde (kalt/caldo [ital.]), Homografen (Ténor/Tenór) u. a.m. Zu einer weiteren Rubrik gehören Ausdrücke, die orthografisch, lautlich und/oder semantisch ähnlich sind sowie oft identische oder ähnliche morphologische Wurzeln aufweisen. Diese können im Sprachgebrauch zu Unsicherheiten führen, die in der Folge bei der Wahl des kontextuell angemessenen Ausdrucks immer wieder zu Verwechslungen führen. Solche Ausdrücke sind Paronyme, wie z. B.: konzeptuell/konzeptionell, kindisch/kindlich/kindhaft, formal/formell/ förmlich, Methode/Methodik/Methodologie. Sie verursachen gleichermaßen sprachliche Zweifel sowohl unter Fremdsprachenlernenden als auch bei Muttersprachler/innen. Die zugrunde liegenden Schwierigkeiten lassen sich auf unterschiedliche Ursachen zurück- 
führen, sie sind auch abhängig von der Art der Paronyme, da diese selbst keine homogene Gruppe darstellen. ${ }^{1}$ Sich dem Phänomen der Paronymie aus empirischer, sprachgebrauchsorientierter Perspektive zu nähern, ist das Ziel des Projektes „Paronymwörterbuch“ am Institut für Deutsche Sprache in Mannheim. Im Zentrum stehen korpusgestützte lexikologische Neubetrachtungen und die lexikografische Dokumentation dieser Ausdrücke in einem innovativen, multifunktionalen Online-Nachschlagewerk. Einerseits wurde Paronymie bisher nur aus strukturalistischer, und nicht aus sprachgebrauchsorientierter Perspektive betrachtet. Andererseits fassen existierende einschlägige Wörterbücher den Untersuchungsgegenstand deutlich weiter (z. B. Pollmann/Wolk 2010; Müller 1973), sind nicht auf empirischer Datenbasis erarbeitet sowie traditionell präskriptiv angelegt oder stellen keine aktuellen Dokumentationen der Gegenwartssprache mehr dar. Genau diese bestehenden Defizite sollen behoben und entsprechende Forschungslücken geschlossen werden. Das vorliegende Heft zeigt wichtige Diskussionen und Ergebnisse des Projektes der vergangenen Jahre.

\section{Paronymie: Definition und Klassifikation}

Bei der Paronymie handelt es sich nicht um ein einheitliches Phänomen. Bisherige, traditionelle Klassifizierungen orientierten sich formalistisch an distinktiven Merkmalen. Auch Definitionen zur Paronymie unterlagen strukturalistischen, sprachsystematischen Vorstellungen und basierten auf atomistischen und oftmals kontextunabhängigen Analysen. Vorwiegend aus fremdsprachlicher und sprachdidaktischer Sicht liegt eine detaillierte Klassifikation vor, die uns Einblicke in strukturelle, vor allem morphologische Schemata für die Einordnung von Paronymen liefert (vgl. Lăzărescu 1995, 1999). Die dort vorliegenden Ansätze interpretieren Paronymie als sprachliche Verwechslung. Diese werden präskriptiv als Fehler aufgefasst, welche auf unzureichende Sprachkenntnisse zurückzuführen sind. Normative Sprachbetrachtungen ordneten das Phänomen der Paronymie daher als semantische Normentgleisungen ein. Um Erklärungen für das mögliche Zustandekommen bzw. die vielfältigen Ursachen von Verwechslungen, für kontextuelle Bedingungen, außersprachliche Faktoren sowie deren Einfluss auf Sprachwandel bemühten sich diese Studien nicht. Diese rein präskriptiv ausgerichtete Betrachtungsweise würde so manche heute gängige Paronymverwendung noch als sprachliche Fehler einordnen.

Korpusgestützte und damit sprachgebrauchsorientierte Analysen gab es auf diesem Feld bis zu Beginn des Projektes nicht. Das heißt, mit der Paronymie existierte ein linguistisches Phänomen, dem nach der pragmatischen Wende keinerlei Aufmerksamkeit zuteilwurde und das bisher auch nicht im authentischen Sprachgebrauch empirisch untersucht worden war. Nach Beginn korpusanalytischer Studien wurde schnell klar, dass wir es bei der Paronymie nicht mit einem Randphänomen zu tun haben. Jede Form der Definition, der Kategorisierung oder Einbettung in semantische Modelle bedeutet notwendigerweise, dass die Erklärung von Verwechslungspotenzial, die Beschreibung kontextbedingter Faktoren, das Erfassen kognitiver Aspekte sowie diskursiver Funktionen einschließen und über einen rein morphologischen Ansatz hinausgehen muss. Diesen neuen Verortungen widmet sich der Beitrag von Ruth M. Mell/Ulrich Schnörch/Petra Storjohann im vorliegenden Heft. Sie erläutern, welche Kategorien und Parameter bei der Bestimmung von Paronymgruppen nötig sind und welche Unterschiede zwischen allgemeinsprachlichen

Siehe dazu den Beitrag von Ruth M. Mell/Ulrich Schnörch/Petra Storjohann in diesem Heft. 
und fachsprachlichen Paronymen existieren. Im Zusammenhang damit stehen Fragen darüber, welches Wissen bzw. welche Aushandlungsprozesse eine Rolle dabei spielen. Korpuslinguistische Auswertungen des Gebrauchs von Paronymen zeigen eine Vielfalt an Ausprägungen. Es besteht heute kein Zweifel daran, dass Paronymie kein rein lexikalisches Phänomen ist. Vielmehr sind darüber hinaus auch Weltwissen, Erfahrung sowie bestimmte außersprachliche Komponenten in jenen Situationen von Bedeutung, in denen es zu Verwechslungen oder zu sprachlicher Anpassung kommt. Paronymie existiert nur unter bestimmten linguistischen Bedingungen, kognitiven Voraussetzungen und außersprachlichen Bezügen. Folglich sind Klassifikationskriterien nötig, die sich an textuellen und diskursiven Funktionen und konzeptuellen Wissenskategorien sowie -strukturen und auch an kommunikativer Erfahrung orientieren.

\section{Sprachgebrauch, Sprachwandel und deskriptiver Ansatz}

Die Korpuslinguistik änderte den Blick hin zu großen empirisch ausgerichteten Analysen des Sprachgebrauchs und führte zu deskriptiven Beschreibungen sprachlicher Strukturen. Aber auch neuere kognitive Modelle hinterfragen stärker, wie sprachliche Strukturen und Funktionen im mentalen Lexikon miteinander vernetzt und gespeichert sind. Sprachliche Unsicherheiten entstehen aber nicht nur dort, wo sich Ausdrücke in lautlicher Hinsicht ähneln, sondern vor allem dort, wo eine gewisse semantische Vagheit bei den bezeichneten Konzepten in der Kommunikation besteht. Paronymie ist daher vor allem ein kognitives Phänomen, für das bisher keinerlei kognitive Einbettung im Sinn einer Erklärung und einer theoretischen Verortung erfolgte. Die Neubetrachtung von Paronymen aus kognitiver als auch aus korpuslinguistischer Perspektive wirft zugleich ein Licht auf zahlreiche neue Fragen, die u. a. mit sprachlicher Verwechslung, Prinzipien des Bedeutungswandels sowie konzeptueller Nähe u. Ä. zu tun haben und zeigt Diskrepanzen zur bisherigen, systemorientierten Forschung auf (siehe Storjohann/Schnörch 2017). Eine genauere Vorstellung darüber, was genau unter Paronymie zu fassen ist, kann daher nur nach umfangreichen empirischen Untersuchungen erfolgen. Das Projekt versucht genau hier einen Vorstoß $\mathrm{zu}$ leisten und die Vorstellungen von sprachlicher Verwechslung neu zu justieren. Dies geschieht aus korpuslinguistischer Sicht mit kognitiv ausgerichteten Fragestellungen. Umfangreiche Korpusanalysen schließen deskriptive Beschreibungen des Beobachtbaren ein. Diese werden genutzt, um Tendenzen und aktuelle Sprachentwicklungen aufzuspüren, um Prinzipien semantischer Veränderung und damit einhergehend Einflüsse und Grade sprachlicher Konventionalisierung aufzudecken. Verwechslungen, insbesondere beginnende, sich etablierende und verbreitende „Fehlverwendungen“ können Auslöser von neuen semantischen Aushandlungsprozessen sein. Diese wiederum unterliegen komplexen Einflussfaktoren. Nicht selten sind Paronyme ähnliche Ausdrücke, die aufgrund ihres Verwechslungspotenzials einer gewissen Rivalität unterliegen und damit Impulse zu Annäherung oder veränderten Konzeptualisierungen geben. So nähern sich manche Ausdrücke semantisch einander an, wie im Fall sensibel/sensitiv zu beobachten ist (vgl. Storjohann 2015). Diese Tendenz besteht eher bei hochpolysemen Ausdrücken, die zusätzlich metaphorischen und metonymischen Verschiebungen unterliegen. Andere, eher monoseme Ausdrücke mit eindeutiger „Bezeichnungskraft“ sind weniger bis gar nicht neu aushandelbar, weil sie eine Lücke in der Konzeptualisierung hinterlassen würden, die unbesetzt bliebe (z. B. konkav/konvex). Rivalität zwischen Paronymen gibt es aber auch zwischen alltagssprachlicher und fachsprachlicher Verwendung (z. B. Parodontose/Parodontitis). Dazwischen gibt es Fälle, bei denen neue Aushandlungsprozesse zu beobachten 
sind, bei denen sich aber erst im Laufe der Zeit zeigen muss, was sich davon konventionalisiert und im Sprachgebrauch durchgesetzt haben wird.

Während im Falle der Paronymie Ursachenforschung und Veränderungen im Bereich sprachlicher Unsicherheiten nicht im Fokus strukturalistischer Studien standen, werden heute kognitiv verankerte und konzeptuell veränderbare Konstellationen bei der Analyse einbezogen. Dazu sind kontextuelle Untersuchungen von tatsächlichem Sprachgebrauch in verschiedensten kommunikativen Situationen nötig, um aus Sprachmustern Faktoren abzuleiten, die die Wahl bestimmter Ausdrücke favorisieren. Diese werden unter Umständen in der Wahl lexikalischer Sequenzen sichtbar. Korpusdaten und systematische Zugänge zu sprachlichen Massendaten ermöglichen die Unterscheidung zwischen weniger oder stärker konventionalisierten Strukturen. Auf diese Weise lässt sich herausfinden, welche Ausdrücke tatsächlich und in welchem Umfang im Sprachgebrauch verwechselt werden. Semantische Gemeinsamkeiten bzw. Unterschiede können gezielt mit explorativen kontrastiven Verfahren ermittelt werden. Dies geschieht durch Untersuchungen von Kollokationsprofilen, wie sie sich über verschiedene Zeiträume abzeichnen. Dadurch lassen sich bestimmte Strukturen und Aushandlungsprozesse rekonstruieren, zudem können Prinzipien sichtbar werden, die semantischen Wandel begünstigen. Einige dieser Aspekte können heute bereits besser erfasst und beschrieben werden. Andere werden künftig im Projekt stärker im Vordergrund stehen. Interessierte werden für Bedeutungswandel und lexikalische Variation sensibilisiert, indem das Wörterbuch auch Erklärungsansätze und diverse Einflussfaktoren in die deskriptive Beschreibung miteinfließen lässt.

\section{Korpora, Methoden und Verfahren: von Stichwortermittlung bis Bedeutungsanalyse}

Seit drei Jahren werden Paronyme erstmals im öffentlichen Sprachgebrauch und unter extensivem Einsatz korpuslinguistischer Methoden untersucht, sowohl bewährter als auch neuer explorativ kontrastiver. Diese Arbeit brachte zahlreiche Erkenntnisse und Einsichten in das Verwechslungspotenzial von ähnlich lautenden und semantisch nahen Ausdrücken. Korpusdaten ${ }^{2}$ waren auch die Grundlage zur Ermittlung von Paronymkandidaten. In einem ersten Schritt wurden die typischsten Muster ermittelt, wie z. B. -frei/-los (kostenfrei/kostenlos), -haft/-lich (jugendhaft/jugendlich), -isch/-onisch (elektrisch/elektronisch), -ung/-samkeit (Bedeutung/Bedeutsamkeit), die bei Paronympaaren vorkommen. Über 150 solcher Muster waren die Grundlage dafür, Kandidaten aus den Korpora aufzuspüren. Rein formal stimmen diese Paare in allen Zeichen überein, besitzen jedoch jeweils einen invarianten Teil, der sich häufig - jedoch nicht zwangsläufig - als Suffix oder seltener Präfix einordnen lässt. Über 9.000 potenzielle Fälle wurden korpusgestützt extrahiert und anschließend in einem Triple-Blind-Verfahren redaktionell ausgewertet. 2.000 davon qualifizierten sich als Stichwortkandidaten. Darunter fallen Simplizia wie auch Komposita, die in Haupt- und Nebeneinträge eingeordnet wurden (z. B. Technik/Technologie, analog dazu: Informationstechnik/Informationstechnologie, Computertechnik/Computertechnologie). Eine manuelle Auswertung der Ergebnisse war nötig, da sich aufgrund des automatisierten Verfahrens oder von Fehllemmatisierungen auch Fehler einschlichen. So wurden Fälle wie Metropole/Metropolis oder Synopse/Synopsis mit dem Muster -e/-is aufgenom-

2 Das Deutsche Referenzkorpus (DeReKo) war die Datenbasis für die systematische Ermittlung potenzieller Paronympaare, die in Zusammenarbeit mit dem Programmbereich „Korpuslinguistik“ am IDS entstand (siehe auch www l.ids-mannheim.de/kl/projekte/korpora; Stand: 26.6.2018). 
men, andere, wie Türke/Türkis, Dose/Dosis wurden „händisch“ aussortiert. Analog konnten Paare wie posen/posieren, blocken/blockieren (-en/-ieren) aufgenommen werden, aber Regen/Regieren stellt hingegen kein Paronympaar dar. Peripher und peripheral (-/-al) sind als Kandidaten aufzunehmen, Termin/Terminal nicht. Ob es sich tatsächlich um verwechselbare Ausdrücke im Sinne der Paronymie handelt, kann i.d.R. erst nach einer semantischen Analyse festgestellt werden. Alle auf diese Weise gewonnenen, vorab ausgewerteten potenziellen Paronympaare/-gruppen (ca. 260 Haupteinträge) wurden anschließend mit einer Kennzahl für die Korpushäufigkeit indiziert, um Aussagen über ihr Vorkommen zu treffen. ${ }^{3}$ Ein Blick in die umfangreiche Stichwortliste verrät schnell, dass Paronymie entgegen allgemeiner Vorstellungen keinesfalls ein Randphänomen ist.

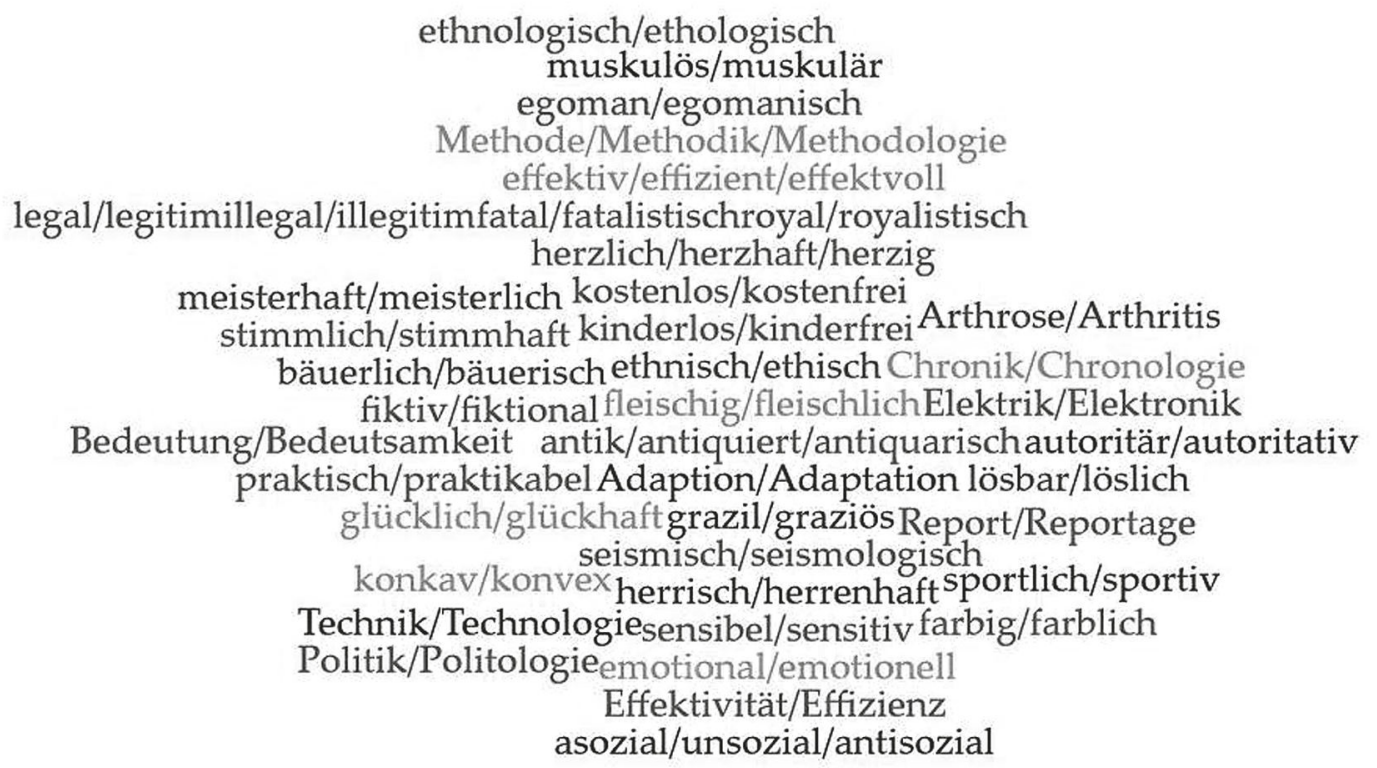

Abb. 1: Wortwolke mit ausgewählten Paronymen, erstellt mit Wortwolken-Generator (Online: www.wortwolken.com; Stand: 26.6.2018)

Man findet zahlreiche standardsprachliche Wortpaare, aber auch viele fachsprachliche bzw. domänenspezifische Paronyme unter den Kandidaten. Der Großteil sind Adjektive, aber auch Nomen und Verben kommen vor. Die Spanne reicht von hochfrequenten bis hin zu selten vorkommenden Paaren. Dass dabei kein regelhaft fassbarer Gebrauch festgestellt werden kann, wie es die Wortbildungsregeln mit den beteiligten Suffixen nahelegen würden, zeigt Stefanie Reckenthäler anhand des Beispiels von zehn Adjektivpaaren, die mithilfe des Suffixes -frei/-los (z. B .: kinderlos/kinderfrei, wertfrei-wertlos) gebildet werden.

Mithilfe korpuslinguistischer Verfahren wurden aber nicht nur potenzielle Kandidaten erfasst. Diese können auf synchroner wie auch auf mikrodiachroner bis hin zur historischen Ebene systematisch nach typischen Gebrauchskontexten geordnet und hinsichtlich ihrer Verwendungen redaktionell seziert werden, um auf diese Weise zentrale Bedeutungsaspekte sowie kleinste Veränderungen aufzuspüren. Zu diesem Zweck wurde auf der Basis von DeReKo ein umfangreiches Korpus aufgebaut, das überregionale Texte einbindet,

3 Eine detaillierte Darstellung zur Gewinnung und Auswertung von Stichwortlisten hat Schnörch (2015) vorgenommen. 
aber auch regionalen Besonderheiten Rechnung trägt. Es umfasst 2,38 Milliarden Textwörter aus 27 verschiedenen Textquellen aus den Jahren 1995-2015 mit unterschiedlichen Gewichtungen und Proportionen. Das Korpus enthält ausschließlich schriftsprachliche Texte aus dem journalistischen Genre. Diese spiegeln in besonderem Maße die öffentliche Gebrauchssprache wider und sie beinhalten höchst unterschiedliche Textsorten (z.B. Wetterbericht, Feuilleton, Anzeigen, Leserbriefe und Meinungen) sowie Berichte aus unterschiedlichsten Themenbereichen (Politik, Wirtschaft, Bildung, Sport, Kunst, Kultur, Literatur). Zeitungen und Zeitschriften spielen medial eine große Rolle, da sie Sprache in redigierter Form mit konventionalisierten, gut etablierten sprachlichen Verwendungen nutzen. Diese sprachlichen Konventionen fließen wiederum in den öffentlichen Sprachgebrauch zurück, weil derartige Publikationen von einer großen Leserschaft rezipiert werden. Das Besondere am Paronymkorpus ist allerdings seine uneingeschränkte Zugänglichkeit über COSMAS II. ${ }^{4}$ Die gesamte redaktionelle, lexikografische Aufbereitung, die Dokumentation sowie die gesamten Dateninterpretationen werden nachvollziehbar und transparent. Die Analysen und Auswertungen konzentrieren sich derzeit allein auf schriftsprachlichen Sprachgebrauch und die Ergebnisse, die in Form von Wörterbucheinträgen sichtbar sind, müssen in Beziehung zur Datengrundlage gesetzt werden. Auch das gelingt mit einer kostenlosen und öffentlich zugänglichen Arbeitsgrundlage besser. ${ }^{5}$

Paronyme in Massendaten strukturiert in ihrem Bedeutungspotenzial erfassen zu können, bedeutet, mit explorativen korpuslinguistischen Methoden und Verfahren an sie heranzutreten, um Konventionalisierungen auf den Grund zu gehen, aufkeimende neue Verwendungen aufzuspüren, ältere verschwinden zu sehen und vor allem kontrastiv zwei Wörter gleichzeitig in ihren Kontexten miteinander vergleichen zu können. Mit diversen Korpusverfahren können wir sehr strukturiert bei der Bedeutungsanalyse vorgehen, verlässliche Aussagen zu prototypischen Mustern oder singulären Erscheinungen treffen und semantische Nuancen identifizieren, die mit subjektiver Introspektion nicht erkennbar sind. Zwei komplementäre Methoden, die Kollokationsanalyse (Belica 1995) und das kontrastive Verfahren (CNS), das auf der Basis von selbstorganisierenden Merkmalskarten (SOM) arbeitet (siehe Belica $2001 \mathrm{ff}$, 2006, 2011), werden im Projekt bei der Analyse von Paronymen eingesetzt. ${ }^{6}$ Die kartografischen Verfahren werden komplementär zu traditionellen Kollokationsanalysen im Projekt genutzt, da einzig mit ihnen Wortpaare mit semantischer Nähe gezielt kontrastiv in ihren kontextuellen Verwendungen und Profilen miteinander verglichen werden können. ${ }^{7}$ Die Beiträge von Ulrich Schnörch und Mareike Teichmann skizzieren beide Methoden und veranschaulichen am konkreten Beispiel ihre Vor-, aber auch ihre Nachteile. Rainer Perkuhn zeigt ergänzend, welche sprachlichen Bezugseinheiten sowie welche Einflussfaktoren bei den verschiedenen Analysemöglichkeiten beachtet werden sollten.

4 Für eine Aufschlüsselung einzelner Korpusbestandteile siehe auch www l.ids-mannheim.de/lexik/paro nymwoerterbuch/dasparonymkorpus.html (Stand: 26.6.2018).

5 Für weitere Pläne, künftig auch andere, z. B. gesprochensprachliche Texte auszuwerten, siehe Abschnitt 7.

$6 \quad$ SOM $=$ Self Organizing Feature Maps, CNS = Contrasting-Near-Synonyms. Diese Verfahren können nur im Zusammenhang mit der Kookkurrenzdatenbank CCDB (Online: http://corpora.ids-mannheim.de/ ccdb; Stand: 26.6.2018) genutzt werden (Belica $2001 \mathrm{ff}$.).

7 Siehe auch den Einsatz der Verfahren z. B. in Marková (2012), Vachková (2010) und Vachková/Belica (2009). 


\section{Foren und Nutzer}

Die Möglichkeiten, Paronyme empirisch, explorativ und kontrastiv neu zu beleuchten, gepaart mit den heutigen technologischen Möglichkeiten innovativer, digitaler und medialer Präsentation, öffnete dem Projekt die Türen für eine neue Ressource, die leicht verwechselbare Wörter in ihrem heutigen Sprachgebrauch paar- bzw. gruppenweise und in ihren individuellen Verwendungsbeziehungen zueinander, dokumentiert. Dass das Interesse an verwechselbaren Ausdrücken groß ist, zeigen Internetforen, in denen Sprecher/ innen Rat in Situationen sprachlicher Schwierigkeiten suchen. Schwierigkeiten bei der Abgrenzung von zwei ähnlichen Ausdrücken resultieren nicht selten in Vermutungen über ,falschen'bzw. ,korrekten' Gebrauch. Neben Synonymen und Fremdwörtern sind es speziell Paronyme, die in allgemeinen Sprachforen kontrovers diskutiert werden. Und es zeigt sich auch, dass sich Muttersprachler/innen der Schwierigkeiten eines angemessenen Gebrauchs von ähnlich klingenden und (partielle) Bedeutungsähnlichkeiten aufweisenden Wörtern bewusst sind. Ein Blick in Internetforen ist lohnend, um herauszufinden, welche konkreten Probleme bei der Verwendung von Paronymen auftauchen, aber auch, um zu erfahren, welche Intuition Sprecher/innen haben, wo sie nachschlagen, wie hilfreich sie diese Informationen finden und ob die angebotenen Wörterbuchinformationen der eigenen Intuition entsprechen oder als widersprüchlich zur sprachlichen Wirklichkeit empfunden werden. ${ }^{8}$ Man erfährt auch, welches sprachliche sowie außersprachliche Wissen bei der Foren-Community vorhanden ist, inwieweit sich Fragende von den Antworten anderer überzeugen lassen und wie normativ die Antworten sind oder sein sollten. Untersuchungen zu Webbeiträgen und Sprachanfragen von Sprachinteressierten aus gemeinschaftlichen Foren sind, wie Beispiel 1 zeigt, aus lexikografischer Sicht höchst aufschlussreich (vgl. Storrer 2013, S. 1251; Storjohann 2016).

Hallo, gerade in den Nachrichten: Westerwelle sagt, Deutschland werde Japan ,technisch und auch technologisch" Hilfe leisten. Hand aufs Herz - wer kann ohne nachzuschlagen sagen, was das konkret bedeutet (und tut es auch)? lg hahu

Antwort 1

Aus dem Stegreif: „technisch“: die Technik betreffend, „technologisch“: die Technologie (Lehre von der Technik) betreffend, In übertragenem Sinn bedeutet es in etwa: „sowohl in praktischer als auch theoretischer Technik". Konkret: Geräte und Leute die die Geräte bedienen einerseits, Ingenieure und Wissenschaftler andererseits.

Antwort 2

Hallo, wie üblich sind das bloss in die Luft geblasene Wortwolken - was wir den Japanern schicken (sollten) sind Suchhunde. Gruss Reinhard

Antwort 3

Ich weiß, Wikipedia widerspricht mir, aber IMHO ist Technologie ein denglisches Blähwort. Technology ist einfach Technik.

8 Ein großer Dank geht an Frau Wiebke Schulte, die dem Projekt eine umfangreiche Datensammlung mit Sprachanfragen und Antworten zu Paronymen aus Foren zur Verfügung gestellt hat. 
Antwort 4:

Hi, nach dem Wörterbuch der Brüder Grimm: Technologie, f., im 18. jahrh. aus franz. technologie vom griech.-lat. technologia kunst-, gewerblehre, gewerbkunde; davon das adj. technologisch. und:

Technik, f. im 18. jh. aufgenommen aus franz. technique vom griech.-lat. technica (nämlich ars), die kunst-oder gewerbsthätigkeit und der inbegriff der erfahrungen, regeln, grundsätze und handgriffe, nach denen bei ausübung einer kunst oder eines gewerbes verfahren wird. www.dwb.uni-trier.de (Stand: 26.6.2018)

Antwort 5

Moin, Bellawa, Technology ist einfach Technik. schtümmt, gefragt war aber nach Technologie. Und dass den Japanern mit Technologie immoment nicht geholfen ist, schtümmt natürlich auch, aber woher soll ein Außenminister (oder sein Ghostwriter) das denn wissen.

Antwort 6

Technik - die greifbare „Hardware“ (Anlagen, Maschinen, ),

Technologie - das Wissen und Können, die Technik sinnvoll und sachgerecht einzusetzen.

Viele Grüße, Lutz

Beispiel 1: Anfrage und Antworten aus einem Sprachforum (Online: www.wer-weiss-was.de/t/technischund-technologisch/6359265/6; Stand: 26.6.2018)

Nicht selten bleiben gepostete Fragen trotz zahlreicher Kommentare und Antworten offen oder enden mit der Frage nach Empfehlungen für Nachschlagewerke, die als zuverlässige Quellen bewertet werden. Dies wiederum zeigt uns, wie groß das Bedürfnis nach verlässlichen, detaillierten und punktuellen Informationen ist. Für die Konzeption der neuen Online-Ressource „Paronyme - Dynamisch im Kontrast“ wurden Einträge aus Foren ausgewertet, um Nutzer/innen und ihre Bedürfnisse in den Mittelpunkt zu rücken. So war es uns möglich, zahlreiche konkrete Aspekte, die sprachlichen Zweifel verursachen, genauer zu identifizieren sowie das allgemeine Nachschlageverhalten von Fragenden untersuchen zu können. Dies geschah vor dem Hintergrund, geeignete und vor allem hilfreiche Beschreibungsformen und Navigationsstrukturen für das neue Wörterbuch zu finden. So wissen wir, dass die Antworten auf Sprachanfragen ausreichend kontextuelle Informationen bereitstellen müssen, um zu überzeugen. Sie sollten deskriptiv ausreichend fundiert, gleichzeitig präzise und auf einen schnellen Blick erkennbar sein. Nur wenn diese Bedingungen erfüllt sind, kehren Nutzer/innen immer wieder zum Paronymwörterbuch zurück, ohne irritiert die Seite zu verlassen und in Foren zu stöbern.

\section{Ein neues kognitiv-orientiertes Wörterbuch}

Ein Ziel des Projektes ist es, ein neues digitales Wörterbuch ${ }^{9}$ zu schreiben, das nach Zuverlässigkeit strebt und diese auf folgendem Weg zu erreichen sucht: Auf der Grundlage authentischer Sprachdaten werden grundlegende Gemeinsamkeiten und Unterschiede von Paronymen dokumentarisch herausgearbeitet. Dabei wird den Ansprüchen moderner Mediennutzung durch Smartphones oder Tablets Rechnung getragen. Auch die hypertextuellen Möglichkeiten der Internetlexikografie werden deutlich stärker als bisher wahrge-

9 Das neue Paronymwörterbuch „Paronyme - Dynamisch im Kontrast“ ist kostenlos über die Plattform OWID ${ }^{\text {plus }}$ aufrufbar, siehe auch www.owid.de/plus (Stand: 26.6.2018). 
nommen. Während in den letzten beiden Dekaden korpuslinguistische Theorien Spuren in der allgemeinen Lexikografie hinterließen, spiegeln sich bis heute kaum kognitiv-semantische Vorstellungen in Wörterbüchern wider (vgl. Ostermann 2015). Schon seit Längerem werden Forderungen nach Einflussnahme von semantischer Theorie auf die Wörterbucharbeit (z. B. Gries 2006; Rundell 2012) laut. Daher wurden konzeptuell ausgerichtete Angaben, wie sie bisher in gängigen Wörterbüchern fehlten, integriert und werden im Beitrag von Petra Storjohann in diesem Heft erläutert. Eine stärkere kognitive Ausrichtung der Angaben kann gewährleisten, dass linguistische und enzyklopädische Informationen, aber auch sprachliches Wissen basierend auf Erfahrung und ontologischer Kategorisierung zusammen präsentiert werden können. Mit diesem Ergebnis ist es möglich, in viel größerem Maße auf die Bedürfnisse der Nutzer/innen einzugehen. Der Beitrag zu diesem Thema ist daher auch ein eindringliches Plädoyer für die Kognitive E-Lexikografie.

Das neue Wörterbuch „Paronyme - Dynamisch im Kontrast“ stellt einen Bruch mit traditionellen, linearen und starren Einträgen dar. Um unsere Forschungsergebnisse angemessen in einem Wörterbuch darzustellen und verschiedenen Nutzerbedürfnissen gerecht zu werden, bedarf es flexibler Darstellungsformen bzw. Beschreibungsebenen. Die stärkere Ausschöpfung text-technologischer und hypertextueller Möglichkeiten steht im Zentrum der lexikografischen Präsentation. Lexikografische Informationen können in OnlineAnwendungen informationsspezifisch flexibilisiert und Inhalte interessenspezifisch dynamisch generiert werden. Menüoptionen bestimmen, auf welche Weise Wörterbuchangaben zusammengefasst und präsentiert werden: Folglich ändert sich daraufhin auch der jeweilige Fokus auf ein Phänomen (z. B. Anordnung nach Frequenz, Gemeinsamkeiten, Unterschieden, Kollokationsprofil, aber auch Änderung der Reihenfolge der Paronymwörter bei größeren Gruppen). Ein- und ausblendbare Elemente begrenzen oder erweitern die Informationen zu kontextuellen Verwendungen. Spezielle Menüoptionen ermöglichen verschiedenartige dynamisch aufrufbare Anordnungsmodi der Inhalte und bieten zudem interaktive Visualisierungen an. Die Nachschlagenden können selbst entscheiden, in welcher Form bestimmte Informationen dargestellt bzw. angeordnet werden. Das Nachschlagewerk geht damit einen entscheidenden Schritt weg vom statischen Wörterbuch hin zur dynamischen, multifunktionalen Online-Ressource.

\section{Ausblick}

In den vergangenen drei Jahren lag der Schwerpunkt des Projektes auf der Ermittlung, Analyse und Dokumentation kontextueller Verwendung von Paronymen in der öffentlichen Gebrauchssprache, so wie sie in der Schriftsprache erkennbar ist. In den nächsten Jahren sollen weitere Forschungslücken geschlossen bzw. die lexikografische Dokumentation vervollständigt werden. Das schließt die Untersuchung gesprochensprachlicher bzw. quasi-spontansprachlicher Materialien ein, um das Bild von paronymem Gebrauch abzurunden. Eine inhaltliche Erweiterung in drei Bereiche ist geplant: Erstens soll das Wörterbuch um Informationen zu fachsprachlichen Paronymen ergänzt werden, da unter den Stichwörtern zahlreiche fachsprachliche Ausdrücke sind und diese in ihren jeweiligen Fachsprachen teilweise anders als in alltäglicher Kommunikation verwendet werden. Zweitens liegen bei einigen Wortpaaren Abweichungen zwischen Verwendungen in der Schriftsprache und in mündlicher Kommunikation vor. In letzterer sind Entwicklungstendenzen zum Teil sichtbarer, da diese Texte spontan und intuitiver als redigierte Zeitungs- 
berichte entstehen. Daher werden künftig Auswertungen gesprächslinguistischer Ressourcen eine Rolle spielen. Und drittens wird das Wörterbuch um Details zu Tendenzen und zu Wandelprozessen erweitert, insbesondere für solche Paare, bei denen Veränderungen in jüngerer Vergangenheit nachweisbar sind. Derartige Beschreibungen sollen Nachschlagende stärker für gegenwärtig stattfindende oder kürzlich abgeschlossene Wandelprozesse sensibilisieren.

Die lexikografische Dokumentation neuer Rubriken bedarf gründlicher empirischer Analysen und erlaubt gleichzeitig, weitere Forschungsfragen, die in diesen Zusammenhängen entstehen, zu beantworten. Einzelstudien zu Paronymen in mündlicher und fachsprachlicher Kommunikation sowie solche, die Prinzipien von Bedeutungswandel aufspüren, werden folgen, um verbleibende Fragen zu klären, die helfen, unser bisheriges Bild lexikalisch-konzeptueller Beziehungen, wie der Paronymie, in vielseitigen Gebrauchssituationen abzurunden.

\section{Literatur}

Belica, Cyril (1995): Statistische Kollokationsanalyse und -clustering. Korpuslinguistische Analysemethode. Mannheim: Institut für Deutsche Sprache. http://corpora.ids-mannheim.de (Stand: 17.6.2014).

Belica, Cyril (2001 ff.): Kookkurrenzdatenbank CCDB (Version 3.3) - Eine korpuslinguistische Denk- und Experimentierplattform für die Erforschung und theoretische Begründung von systemisch-strukturellen Eigenschaften von Kohäsionsrelationen zwischen den Konstituenten des Sprachgebrauchs. Mannheim: Institut für Deutsche Sprache. http://corpora.ids-mannheim.de/ccdb (Stand: 26.6.2018).

Belica, Cyril (2006): Modellierung semantischer Nähe: Kontrastierung von nahen Synonymen. Korpusanalytische Methode. Mannheim: Institut für Deutsche Sprache. http://corpora.ids-mannheim.de/ccdb (Stand: 26.6.2018).

Belica, Cyril (2011): Semantische Nähe als Ähnlichkeit von Kookkurrenzprofilen. In: Abel, Andrea/Zanin, Renata (Hg.): Korpora in Lehre und Forschung. Bozen: Bozen University Press. S. 155-178.

COSMAS II = Corpus Search, Management and Analysis System. Das Portal für die Korpusrecherche in den Textkorpora des Instituts für Deutsche Sprache. Mannheim: Institut für Deutsche Sprache. www.idsmannheim.de/cosmas2 (Stand: 1.11.2017).

DeREKo = Deutsches Referenzkorpus. Mannheim: Institut für Deutsche Sprache. wwwl.ids-mannheim.de/ $\mathrm{kl} /$ projekte/korpora (Stand: 26.6.2018).

Gries, Stefan Thomas (2006): Corpus-based methods and cognitive semantics: The many senses of to run. In: Gries, Stefan Thomas/Stefanowitsch, Anatol (Hg.): Corpora in cognitive linguistics corpus-based approaches to syntax and lexis. Berlin/New York: De Gruyter. S. 57-100.

Làzàrescu, Ioan (1995): Deutsche Paronyme. In: Grazer Linguistische Studien 43. S. 85-93.

Làzàrescu, Ioan (1999): Die Paronymie als lexikalisches Phänomen und die Paronomasie als Stilfigur im Deutschen. Bukarest: Editura Anima.

Marková, Vera (2012): Synonyme unter dem Mikroskop. Eine korpuslinguistische Studie. Tübingen: Narr. (= Korpuslinguistik und interdisziplinäre Perspektiven auf Sprache 2).

Müller, Wolfgang (1973): Leicht verwechselbare Wörter. Mannheim: Bibliographisches Institut. (= DudenTaschenbücher 17).

Ostermann, Carolin (2015): Cognitive lexicography: A new approach to lexicography making use of cognitive semantics. Berlin/Boston: De Gruyter. (= Lexicographica Series Maior 149).

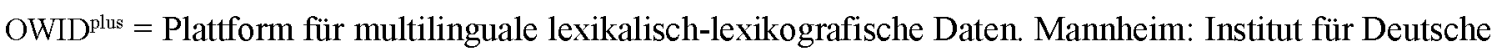
Sprache. www.owid.de/plus (Stand: 26.6.2018). 
Paranymwörterbuch $=$ Paronyme - Dynamisch im Kontrast. http://owid.de/plus, http://www.owid.de/ parowb (Stand: 5.11.2018).

Pollmann, Christoph/Wolk, Ulrike (2010): Wörterbuch der verwechselten Wörter. 1000 Zweifelsfälle verständlich erklärt. Stuttgart: PONS.

Rundell, Michael (2012): It works in practice but will it work in theory? The uneasy relationship between lexicography and matters theoretical. In: Vatvedt Fjeld, Ruth/Torjusen, Julie Matilde (Hg.): Proceedings of the $15^{\text {th }}$ EURALEX Congress. Oslo: University of Oslo. S. 47-92. http://euralex.org/category/publica tions/euralex-oslo-2012 (Stand: 26.6.2018).

Schnörch, Ulrich (2015): Wie viele Paronympaare gibt es eigentlich? Das Zusammenspiel aus korpuslinguistischen und redaktionellen Verfahren zur Ermittlung einer Paronymstichwortliste. In: Sprachreport 31, 4. S. 16-26.

Storjohann, Petra (2015): Was ist der Unterschied zwischen sensitiv und sensibel? In: Zeitschrift für Angewandte Linguistik 62, 1. S. 99-122.

Storjohann, Petra (2016): Vom Interesse am Gebrauch von Paronymen zur Notwendigkeit eines dynamischen Wörterbuchs. In: Sprachreport 32, 4. S. 32-43.

Storjohann, Petra/Schnörch, Ulrich (2017): Sprachlicher Wandel der Gegenwart und seine Dokumentation in einem Wörterbuch. In: Vachková, Marie/Šemelík, Martin/Kloudová, Vera (Hg.): Themenheft Lexikographie, Germanistica Pragensia, AUC Philologica 4. S. 133-172.

Storrer, Angelika (2013): Representing dictionaries in hypertextual form. In: Gouws, Rufus et al. (Hg.): Dictionaries. An international encyclopedia of lexicography. Zusatzbd.: Recent developments with focus on electronic and computational lexicography. Berlin/Boston: De Gruyter. S. 1244-1253.

Vachková, Marie (2010): Zur Erforschung und Erfassung der diskursgebundenen semantischen Kontraste auf der Grundlage des SOM-Modells. In: Tématické číslo Germanistica Pragensia 20. S. 193-208.

Vachková, Marie/Belica, Cyril (2009): Self-organizing lexical feature maps. Semiotic interpretation and possible application in lexicography. In: Interdisciplinary Journal for Germanic Linguistics and Semiotic Analysis 13, 2. S. 223-260.

Dr. Petra Storjohann

Institut für Deutsche Sprache Mannheim

R5, 6-13

68161 Mannheim

E-Mail: storjohann@ids-mannheim.de 